\title{
Signature of Plausible Accreting Supermassive Black Holes in Mrk 261/262 and Mrk 266
}

\author{
Gagik Ter-Kazarian and Lusine Sargsyan \\ Byurakan Astrophysical Observatory, Aragatsotn, 378433 Byurakan, Armenia \\ Correspondence should be addressed to Gagik Ter-Kazarian; gago_50@yahoo.com
}

Received 7 May 2013; Revised 13 July 2013; Accepted 22 August 2013

Academic Editor: William Reach

Copyright (C) 2013 G. Ter-Kazarian and L. Sargsyan. This is an open access article distributed under the Creative Commons Attribution License, which permits unrestricted use, distribution, and reproduction in any medium, provided the original work is properly cited.

\begin{abstract}
We address the neutrino radiation of plausible accreting supermassive black holes closely linking to the 5 nuclear components of galaxy samples of Mrk 261/262 and Mrk 266. We predict a time delay before neutrino emission of the same scale as the age of the Universe. The ultrahigh energy neutrinos are produced in superdense protomatter medium via simple (quark or pionic reactions) or modified URCA processes (G. Gamow was inspired to name the process URCA after the name of a casino in Rio de Janeiro). The resulting neutrino fluxes for quark reactions are ranging from $J_{\nu \varepsilon}^{q} /\left(\varepsilon_{d} \operatorname{erg~cm}^{-2} \mathrm{~s}^{-1} \mathrm{sr}^{-1}\right) \simeq 1.1 \times 10^{-9}$ to $8.2 \times 10^{-9}$, where $\varepsilon_{d} \sim 10^{-12}$ is the opening parameter. For pionic and modified URCA reactions, the fluxes are $J_{\nu \varepsilon}^{\pi} \simeq 0.1 J_{\nu_{\varepsilon}}^{q}$ and $J_{\nu \varepsilon}^{\mathrm{URCA}} \simeq 7.4 \times 10^{-11} J_{\nu \varepsilon}^{q}$, respectively. These fluxes are highly beamed along the plane of accretion disk, peaked at ultrahigh energies, and collimated in smaller opening angle $\theta \sim \varepsilon_{d}$.
\end{abstract}

\section{Introduction}

The interactions between galaxies may promote key processes in galaxy evolution including mass assembly, star formation, morphological transformation, and activity of AGNs. Among various proposals is the hierarchical galaxy formation hypothesis, wherein the galaxies and galactic structures are formed through merging of smaller galaxies/structures. The galaxy-galaxy interactions/mergers, as well known, can significantly enhance the star formation rate in the respective galaxies; see, for example, [1] for a review. Each proposal towards galaxy-galaxy interactions/mergers has its own advantage and limitations in proving the whole view of the issue. If tidal plume and tails indicative of classical merger remnants can be seen, it seems reasonable to accept the merging hypothesis, as in the case of many Markarian galaxies with a binary nucleus. However, some Markarian galaxies with multiple nuclei do not show any sign of tidal effects. If obvious tidal features are not easily detectable, it is wise to place constraints on the likelihood of the merger hypothesis as it was done in the pioneering discussions of Ambartsumian in the 1950's and 1960's for analyzing the observational data of eruptive activity of galactic nuclei and energetic nonstationary phenomena in the Universe [2-4]. Recently, we present a spectroscopic investigation [5] of the galaxy samples of Mrk 261/262 and Mrk 266. The nuclear components in both of these samples show quite similar emission lines in their spectra. Besides, they do not show any sign of tidal effects. Therefore, one cannot exclude the alternative possibility that galactic nuclei in these samples, for example, may separate by fission rather than merging. A useful framework for such viewpoint is the microscopic theory of black hole (MTBH), originally proposed by [6] (see references therein), and further discussed by [7]. Therefore, our interest is to apply the predictions of MTBH to observational data of unique galaxy samples of Mrk 261/262 and Mrk 266 as a backdrop to validate the microscopic approach with some observational tests. The MTBH supports the idea of accreting supermassive black holes linked to AGNs and their neutrino radiation. With this perspective in sight, we address the essential features of 5 nuclear components in Mrk 261/262 and Mrk 266. Certainly, it would be proper also to compare the prediction on neutrino emission from the large number of AGN black holes, well known from the literature. This will be done elsewhere. 
1.1. Motivation. We have put forward the microscopic theory of black hole as a possible way to innovate the solution to the key problem of the AGN powerhouse. Recall that ever since the discovery of the rapid release of large amounts of energy from galactic nuclei and the origin of compact objects, the main problem has been to identify the physical mechanisms behind these phenomena. A major unsolved problem is how efficiently such huge energies observed can be generated. This energy scale severely challenges the conventional source models. The longstanding standard phenomenological black hole model (PBHM) cannot successfully support such investigations, because the theory breaks down inside the event horizon, whereas the meaningless singularity develops at the center inside of the stationary black hole, which is causally disconnected from the exterior world. The ultimate fate of the collapsing matter once it has crossed the black hole surface is unknown. So, the central singularity does not allow a further mass assembly. In the framework of PBHM, neither a growth of black hole mass promoted by accretion process nor an increase of energy density occur. Therefore, the required huge energy scale to account for the resulting activity of galactic nuclei cannot be reached. On the other hand, a complex study of AGN evolution requires a comprehensive understanding of the important phenomenon of the black hole growth. This idea gathers support from a breakthrough made in recent observational, theoretical, and computational efforts on understanding of coevolution of black holes and their host galaxies, particularly through self-regulated growth and feedback from accretion-powered outflows; see, for example, [8-14]. Thus, the PBHM cannot be currently accepted as the convincing model for addressing the aforementioned problems. To fill the void which the standard PBHM presents, one possible way to improve this situation is the microscopic theory of black hole. The MTBH is the extension of the $\mathrm{PBHM}$, which rather completes $\mathrm{PBHM}$ by exploring the most important processes of rearrangement of vacuum state and a spontaneous breaking of gauge symmetry in gravity at huge energies (see $[6,15,16])$. Thereby, a discussed gravitational theory is consistent with the general relativity up to the limit of neutron stars. Namely, the observational values are in a variety of ways well within the range of theoretically allowed masses of neutron star models discussed here. But MTBH also manifests its virtues applied to the physics of internal structure of galactic nuclei. A significant change of properties of space-time continuum, so-called inner distortion (ID), arises simultaneously with the strong gravity. This causes the matter to undergo a phase transition of second kind, which supply a powerful pathway to form a stable superdense protomatter core (SPC) $[6,7]$ (see Appendix A.1 of present paper). The crucial difference from $\mathrm{PMBH}$ is that the central singularity cannot occur now. Instead, this is replaced by finite though unbelievably extreme conditions. The SPC accommodates the highest energy scale up to hundreds $\mathrm{ZeV}$ in protomatter core to account for the spectral distribution of the resulting radiation of galactic nuclei. External physics of accretion onto black hole in the earlier part of its lifetime has tight links to the processes in Schwarzschild's model. However, an essential difference in the model context between a true black hole and the SPC arises in the later part of its lifetime [7]. The SPC surrounded by the accretion disk presents the microscopic model of AGN (Appendix A.1). The seed black hole might grow up driven by the accretion when it was getting most of its mass. That is, with time an infalling matter forms protomatter disk around protomatter core tapering off faster at reaching out the thin edge of event horizon. At this, metric singularity inevitably disappears and the neutrinos may escape through opened vista to the outside world. We will not be concerned here with the actual details of $\mathrm{MTBH}$. The interested reader is invited to consult the original papers for further details.

1.2. Expected Results. Our computations in the present paper are partially based on [7], which has dealt with the preradiation time (PRT) of neutrinos from AGN black holes with well-determined masses and bolometric luminosities, collected from the literature [17]. The PRT is referred to as the earlier part of the lifetime $T_{\mathrm{BH}}$ of black hole from its birth till neutrino radiation. For the latter part of the lifetime, $T>$ $T_{\mathrm{BH}}$, the black hole no longer holds as a region of space time that cannot communicate with the external Universe (see Appendix A.1). The ultrahigh energy (UHE) neutrinos are produced in superdense protomatter medium of SPC via simple (quark or pionic reactions) or modified URCA processes. Consequently, the neutrinos may escape through opened vista to outside world even after the neutrino trapping. We estimate the $M_{\mathrm{BH}}$ masses and bolometric luminosities $L_{\mathrm{bol}}$ in order to compute the PRTs and neutrino fluxes for the Mrk 261/262 and Mrk 266. These galaxy samples can be served as pure UHE neutrino sources. Note that the point-like sources of neutrinos are the most essential ingredients of Z-burst scenario [18-27] of the origin of ultrahigh energy cosmic ray (UHECR) particles. This scenario is the most economical among the various hypotheses, because the neutrinos can penetrate cosmological distances and their trajectories are not deflected by magnetic fields as they are neutral. The Zburst scenario suggests that if $\mathrm{ZeV}$ astrophysical neutrino beam is sufficiently strong it can produce the observed UHECR particles within $100 \mathrm{Mpc}$ by hitting local light relic neutrinos clustered in dark halos. They form such particles through the hadronic $\mathrm{Z}$ (s-channel production) and $\mathrm{W}$ bosons (t-channel production) decays by weak interactions. The discovery of UHE neutrino sources would also clarify the production mechanism of the $\mathrm{GeV}-\mathrm{TeV}$ gamma rays observed on Earth. The AGNs as significant point sources of neutrinos were analyzed, for example, in [26, 28, 29]. While being hard to detect, neutrinos have the advantage of representing unique fingerprints of hadron interactions and, therefore, of the sources of cosmic rays. At present, a numerous reports are available in the literature on expected discovery potentials and sensitivity of experiments to neutrino point-like sources. Currently, operating high energy neutrino telescopes, see, for example, Antares [30, 31], IceCube [32, 33], Baikal [34], JEMEUSO mission [35], the Telescope Array experiment [36], Pierre Auger Observatory [37, 38], and so forth, attempt to detect UHE neutrinos. For example, in [32] it is reported on the first measurement of the atmospheric electron neutrino flux in the energy range between approximately $80 \mathrm{GeV}$ and 
$6 \mathrm{TeV}$. Using data recorded during the first year of operation of IceCube DeepCore low-energy extension, a sample of 1029 events is observed in 281 days of data, of which $496 \pm 66$ (stat) \pm 88 (syst) are estimated to be cascade events, including both electron neutrino and neutral current events. The rest of the sample includes residual backgrounds due to atmospheric muons and charged current interactions of atmospheric muon neutrinos. The flux of the atmospheric electron neutrinos is consistent with models of atmospheric neutrinos in this energy range. This constitutes the first observation of electron neutrinos and neutral current interactions in a very large volume neutrino telescope optimized for the TeV energy range.

This paper is organized as follows. In Section 2 we present the mass and luminosity estimates of 5 nuclear components of Mrk 261/262 and Mrk 266. Section 3 deals with the PRTsimulations for supermassive black holes in the nuclear components. In Section 4 we compute the fluxes of primary UHE neutrinos produced in the superdense protomatter medium. The concluding remarks are presented in Section 5. We will refrain from providing lengthy details of the MTBH and neutrino flux computations. For these, the reader is referred to Appendices A and B.

\section{The Mass and Luminosity Estimates from Optical Observations of Mrk 261/262 and Mrk 266}

BH Mass Estimate. The results of observations for Mrk 266 with the Spitzer, Hubble, GALEX, Chandra, and XMMNewton space telescopes are presented in [39]. The relationship between $\mathrm{H}$-band bulge luminosity and the mass of the nuclear black hole [40] (Group 1 Galaxies) provides $M_{\mathrm{BH}}$ estimates of $2.3 \times 10^{8}$ and $2.6 \times 10^{8} M_{\odot}$ for Mrk $266 \mathrm{SW}$ and NE, respectively, where the scatter is 0.3 in log. The luminosity of high ionization, mid-infrared emission lines can also be used to estimate $\mathrm{MBH}$ using relationships derived by [41]. The [Ne V] $14.32 \mu \mathrm{m}$ ([O IV] $25.89 \mu \mathrm{m}$ ) emission line luminosity of $1.58(10.5) \times 10^{41} \mathrm{erg} \mathrm{s}^{-1}$, computed from published high resolution Infrared Spectrograph on board Spitzer Space Telescope (IRS) measurements [42, 43], corresponds to $M_{\mathrm{BH}}=1.7(3.2) \times 10^{8} M_{\odot}$ for the SW nucleus, where the scatter is 0.46 and 0.47 in log. These values are in good agreement with the estimates based on the SW bulge luminosity. However, it should be emphasized that the available IRS data did not have sufficient spectral and spatial resolution to extract $[\mathrm{Ne} \mathrm{V}]$ and [O IV] line fluxes for the NE nucleus. The differences arise from the wide scatter in calibrating $M_{\mathrm{BH}}$ versus luminosity or velocity dispersion. Also, there is no similar infrared spectral observation for Mrk261/262. Therefore, we are going to estimate the black hole masses in the same way from our optical spectra of Mrk261/262 and Mrk 266 [5], using the width of the [OIII] $\lambda 5007$ line. Note that a tight correlation exists for active galaxies between the velocity dispersion $\sigma_{*}$ of the bulge in a galaxy and the mass of its nuclear black hole $M_{\mathrm{BH}}[44,45]$. Moreover, many groups have used the width of the [OIII] $\lambda 5007$ line as a substitute for the stellar velocity dispersion $\sigma_{*}$ of galaxy bulges. So, at

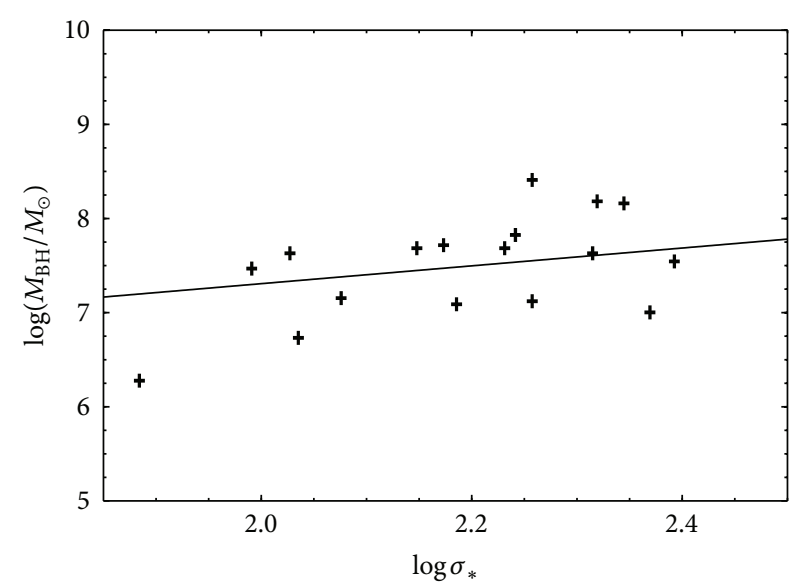

FIGURE 1: The $M_{\mathrm{BH}} / M_{\odot}-\sigma_{*}$ relation on logarithmic scales for 17 objects from [46]. The solid line is the best fits to data of samples.

the very beginning we may plot the $M_{\mathrm{BH}} / M_{\odot}-\sigma_{*}$ relation on logarithmic scales (Figure 1) for 17 objects chosen from [46]. These objects have the most accurate $M_{\mathrm{BH}}$ measurements based on a complete and consistent reanalysis of broad emission-line reverberation-mapping data and [OIII] $\lambda 5007$ FWHMs. Scatter about the mass-luminosity relationship for these AGNs appears to be real and could be correlated with either Eddington ratio or object inclination. The formula of the fitted solid line to the data with a scatter of \pm 0.42 in $\log$ is

$$
\log \left(\frac{M_{\mathrm{BH}}}{M_{\odot}}\right)=2.01( \pm 0.83) \log \sigma_{*}+3.07( \pm 1.83) .
$$

Next, from our optical spectra [5] (Figures 2, 3, and 4) we may calculate the values of $\sigma_{*}$ for Mrk 261/262 and Mrk 266. As a proxy for the velocity dispersion $\sigma_{*}$ we use the [OIII] $\lambda 5007$ line width FWHM $_{[\mathrm{OIII}] / 2.35(\mathrm{~km} / \mathrm{s})}$; see Table 1. And then, from relation (1) we may derive the black hole masses for these values of $\sigma_{*}$; see Table 2. Our optical spectra of the Mrk261/262 are obtained on the $6 \mathrm{~m}$ telescope at Special Astrophysical Observatory of Russia, with the Universal Astronomical Grating Spectrograph. The detector was a 530 $\times 580,18 \times 24 \mu \mathrm{m}$ pixel CCD, and the spectral band was $\lambda(3720 \AA)-\lambda(7150 \AA)$. The hz44 standard star was chosen for flux calibration, and the spectral dispersion was $5.9 \AA /$ pix. The resolution, as measured on the night sky lines, was $\sim 16.3 \AA$ (FWHM). In [5], we also used the observational data of Mrk 266, obtained on the $5 \mathrm{~m}$ Hale telescope at Palomar Observatory with the Norris Spectrograph. Rotating the position angle by 45 degrees, each component was observed in red and blue parts at different position angles of $31^{\circ}, 76^{\circ}$, $121^{\circ}$, and $166^{\circ}$. The pixel scale of detector was $1024 \times 256$ pixels for red part and $180 \times 800$ pixels for blue part. The exposure times amounted to $900 \mathrm{~s}$ for each spectrum in case of position angle $31^{\circ}$, but the $420 \mathrm{~s}$ exposures were taken for the rest of position angles. The spectral dispersions were 2.2 and $0.66 \AA /$ pix, respectively, for spectral bands of blue part, $\lambda(3750 \AA)-\lambda(5500 \AA)$, and red part, $\lambda(6450 \AA)-\lambda(7100 \AA)$. The resolution, as measured on the night sky lines, was $\sim 7.5 \AA$ (FWHM) for the blue part, and $\sim 1.8 \AA$ (FWHM) for 


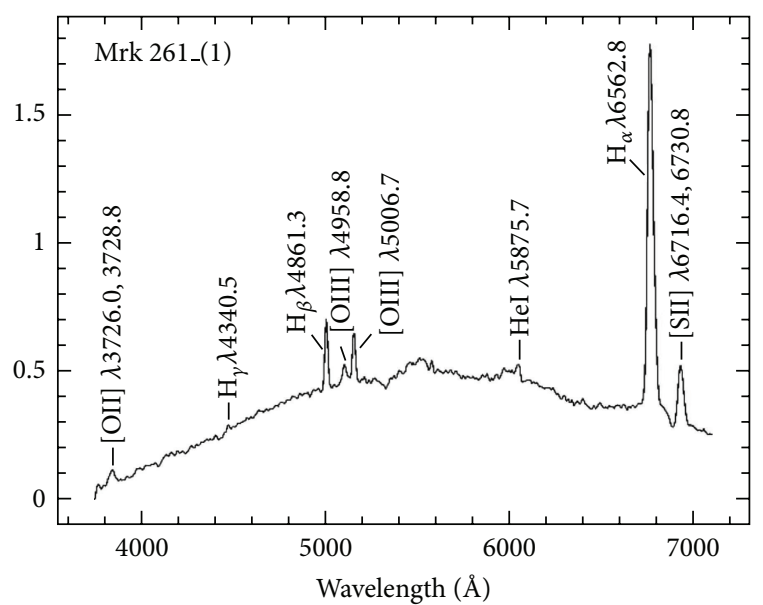

(a)

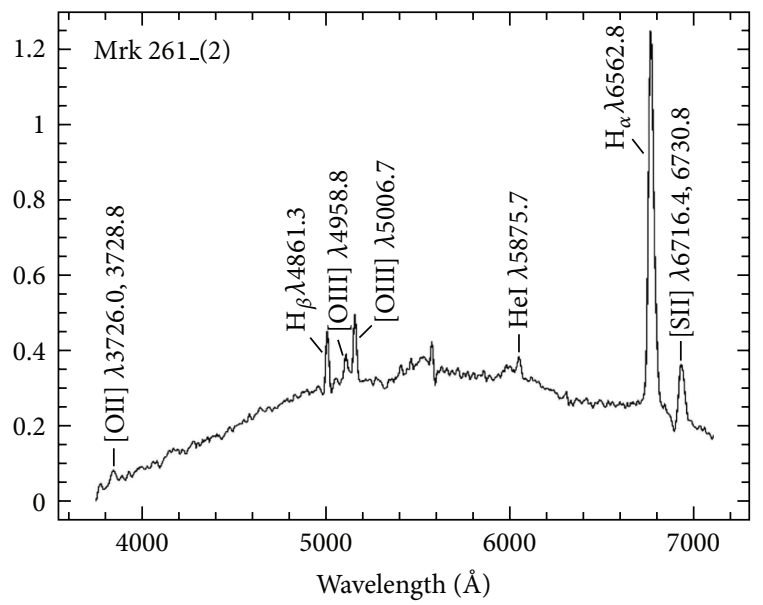

(b)

FIgURE 2: The spectra of the nuclear components Mrk 261_1 and Mrk $261 \_2$ in the sample Mrk 261/262.

the red part. As it is seen, the nuclear components in both of these samples show quite similar emission lines in their spectra. Treating the instrumental profile and the intrinsic profile to sum as Gaussians is a good approximation [47]. A formal solution, in general, is a deconvolution, but this comes very close to the same result. Thus, unfolding wholly the forbidden [OIII] $\lambda 5007$ line profile into its Gaussian component, the question can be resolved by subtraction of instrumental broadening (for the real Doppler broadening $\lambda_{D}$ of the [OIII] $\lambda 5007$ lines see Table 1 ).

Bolometric Luminosity Estimate. The bolometric luminosity is not straightforward to derive for most of the AGNs because of beaming and obscuration. It was sometimes also approximated from optical luminosity, since integration of the spectral energy distribution is usually hampered by lack of wavelength coverage and by variability. In our calculation, we use the total bolometric luminosity, which, according to [48], equals 9.26 times the value of the visual luminosity $\lambda L_{\lambda}(5100 \AA)$. For this, we need to derive the visual luminosities $\lambda L_{\lambda}(5100 \AA)$ for the Mrk 266 and Mrk 251/262. In the first

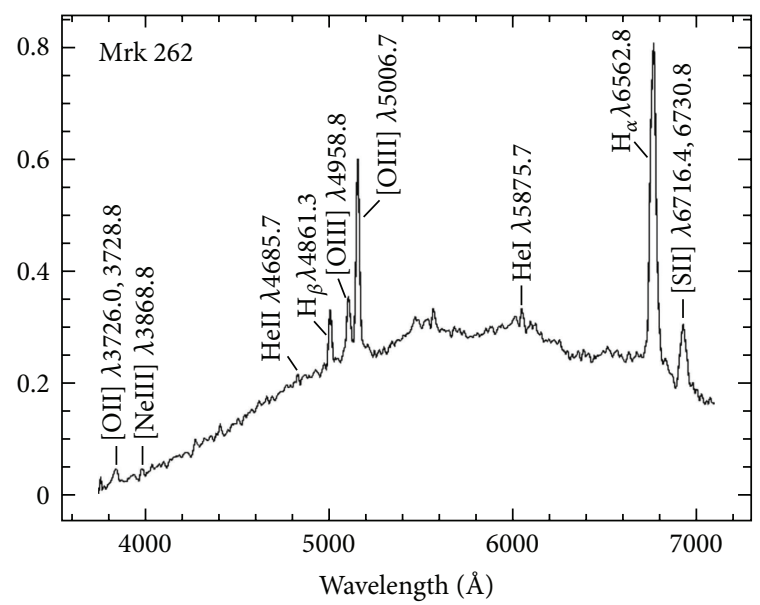

FIgURE 3: The spectrum of the nuclear component Mrk 262 in the sample Mrk 261/262.

TABLE 1: The characteristics of the [OIII] $(\lambda$ 5007) $\mathrm{km} / \mathrm{s}$ line from nuclear spectra in Mrk 261/262 and Mrk 266. Columns: (1) name; (2) redshift; (3) FWHM of the line [OIII] $\lambda 5007$ in $\mathrm{km} / \mathrm{s}$; and (4) $\sigma_{*}=$ FWHM $_{[\mathrm{OIII}] / 2.35(\mathrm{~km} / \mathrm{s})}$ is the width of the [OIII] $\lambda 5007$ line as a proxy for the stellar velocity dispersion $\sigma_{*}$ on logarithmic scale.

\begin{tabular}{lccc}
\hline Name & $z$ & {$[\mathrm{OIII}] \lambda 5007$ FWHM km/s } & $\log \sigma_{*}$ \\
\hline & \multicolumn{3}{c}{ Mrk 261/262 } \\
Mrk261_(1) & 0.0296 & 531 & 2.35 \\
Mrk261_(2) & 0.0301 & 426 & 2.26 \\
Mrk 262 & 0.0304 & 671 & 2.46 \\
\hline \multicolumn{5}{c}{ Mrk 266 } \\
A & 0.02759 & 414 & 2.25 \\
B & 0.02800 & 501 & 2.33 \\
\hline
\end{tabular}

step, we may find a correlation between the visual luminosities $\lambda L_{\lambda}(5100 \AA)$ for 34 AGNs and black hole masses, presented in [46], which is plotted in Figure 5. The fitted line formula is

$$
\begin{aligned}
\log \left(\frac{\lambda L_{\lambda}(5100 \AA)}{L_{\odot}}\right) \\
\quad=1.16( \pm 0.12) \log \left(\frac{M_{\mathrm{BH}}}{M_{\odot}}\right)+1.06( \pm 0.99),
\end{aligned}
$$

with the scatter \pm 0.45 in log. We then looked for the visual luminosities $\lambda L_{\lambda}(5100 \AA)$ for Mrk 266 and Mrk 251/262, which can be estimated from this relation. And then, we derive the required bolometric luminosities; see Table 2 .

\section{PRT Simulations for Mrk 261/262 and Mrk 266}

In the framework of MTBH, the PRT is referred to as the earlier part of the lifetime $T_{\mathrm{BH}}$ of SPC. During this time an infalling matter forms protomatter disk around the protomatter core tapering off faster at reaching out the thin edge of event horizon. That is, $T_{\mathrm{BH}}=M_{d} / \dot{M}$, where $\dot{M}$ is 


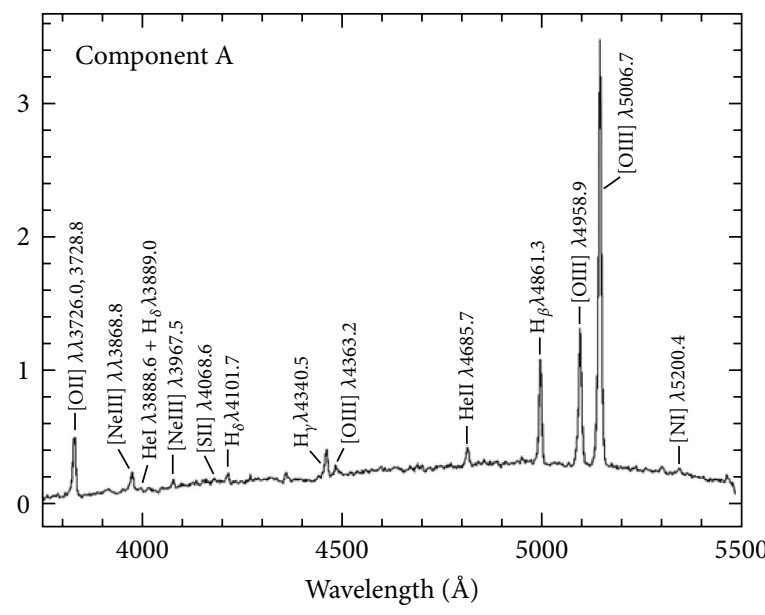

(a)

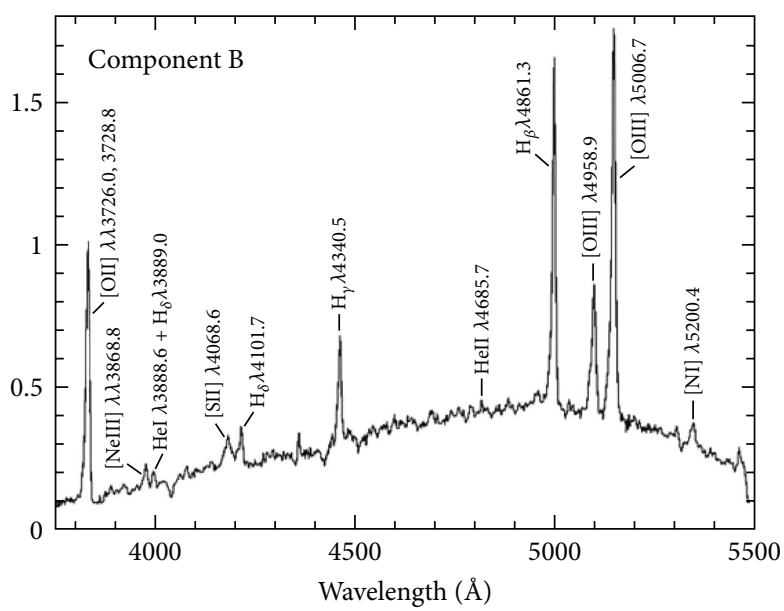

(c)

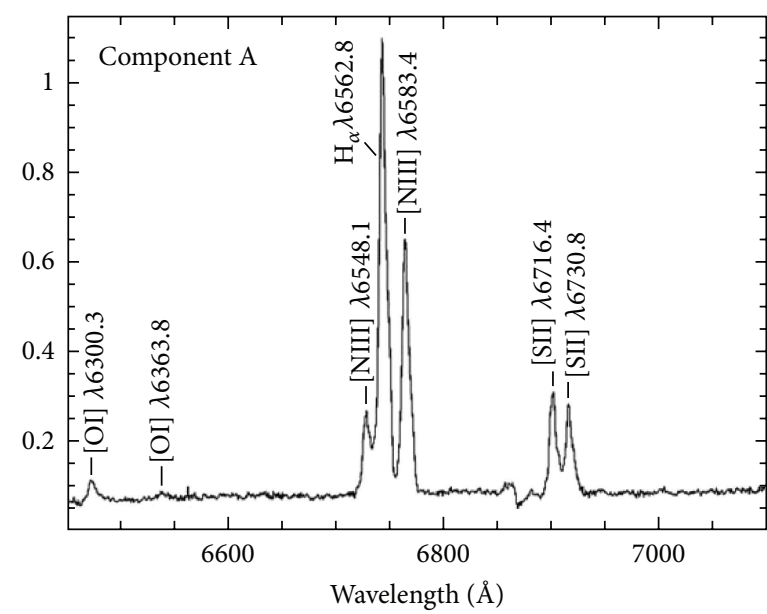

(b)

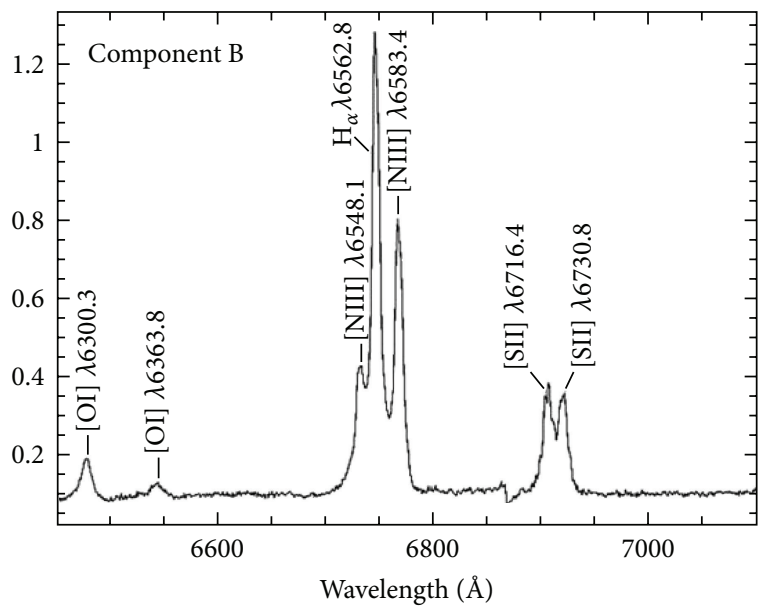

(d)

Figure 4: The blue and red spectra of the nuclear components (A) and (B) in the Mrk 266.

the accretion rate. Assuming a typical mass-energy conversion efficiency of about $\epsilon \sim 10 \%$, in approximation $R_{d} \ll R_{g}$ the PRT reads (see Appendix A.2)

$$
T_{\mathrm{BH}} \simeq 0.32 \frac{R_{d}}{r_{\mathrm{OV}}}\left(\frac{M_{\mathrm{BH}}}{M_{\odot}}\right)^{2} \frac{10^{39} W}{L_{\mathrm{bol}}} \mathrm{yr},
$$

where $r_{\mathrm{OV}}=13.68 \mathrm{~km}$.

SPC-Configurations. As a working model we assume the SPCconfigurations, which are discussed in [6]. They are composed of spherical-symmetric distribution of matter in manyphase stratified states. The I-class SPC-configuration above the density $\rho>\rho_{d}=2.6 \times 10^{16} \mathrm{~g} \mathrm{~cm}^{-3}$ is thought to have central core composed of the $\mathrm{n}$-p-e protomatter, which is mixture of neutrons, protons, and electrons in complete $\beta$ equilibrium, at short nucleon-nucleon distances $r_{\mathrm{NN}} \leq 0.4 \mathrm{fm}$ in presence of ID. The second class SPC-configuration, above the density $\rho>\rho_{d}$, is thought to have protomatter core made of quarks in one bag in complete $\beta$-equilibrium under the
TABLE 2: The physical characteristics of components in Mrk 261/262 and Mrk 266. Columns: (1) name; (2) log of black hole mass; (3) $\log$ of $\lambda L_{\lambda}(5100 \AA) / L_{\odot}$ luminosity; and (4) log of (bolometric luminosity $\left./ L_{\odot}\right)$.

\begin{tabular}{lccc}
\hline Name & $\log \left(M_{\mathrm{BH}} / M_{\odot}\right)$ & $\log \left(\lambda L_{\lambda}(5100 \AA) / L_{\odot}\right)$ & $\log \left(L_{\mathrm{bol}} / L_{\odot}\right)$ \\
\hline & \multicolumn{3}{c}{$\operatorname{Mrk} 261 / 262$} \\
Mrk261_(1) & 7.97 & 10.30 & 11.27 \\
Mrk261_(2) & 7.77 & 10.07 & 11.04 \\
Mrk 262 & 8.18 & 10.55 & 11.51 \\
\hline \multicolumn{4}{c}{ Mrk 266 } \\
A & 7.74 & 10.04 & 11.01 \\
B & 7.91 & 10.24 & 11.21 \\
\hline
\end{tabular}

weak interactions and gluons, including the effects of QCDperturbative interactions (for details see Appendix A.1).

Modeling. With the black hole masses and bolometric luminosities given in Tables 2 and 3, we proceed to PRT-simulations. The main idea comes to solving an inverse problem. 


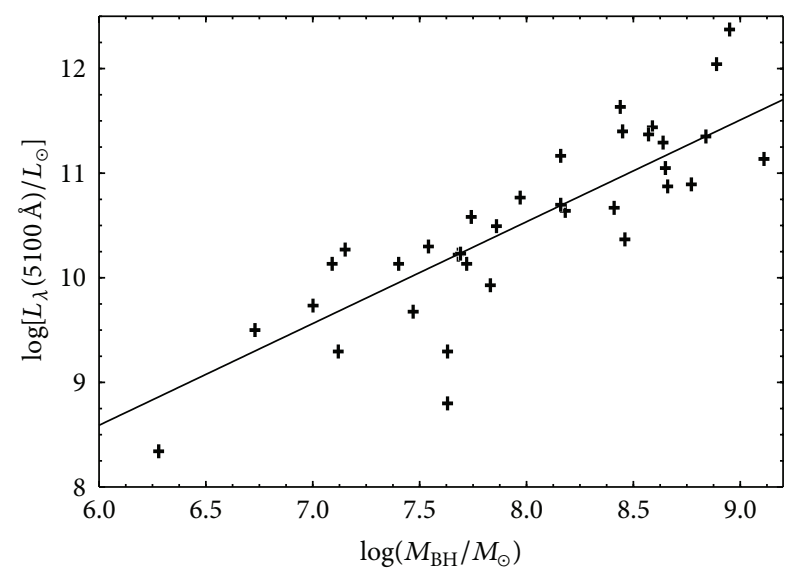

Figure 5: The $M_{\mathrm{BH}} / M_{\odot}-L_{\lambda}(5100 \AA) / L_{\odot}$ relation on logarithmic scales for 34 objects from [46]. The solid line is the best fits to data of samples.

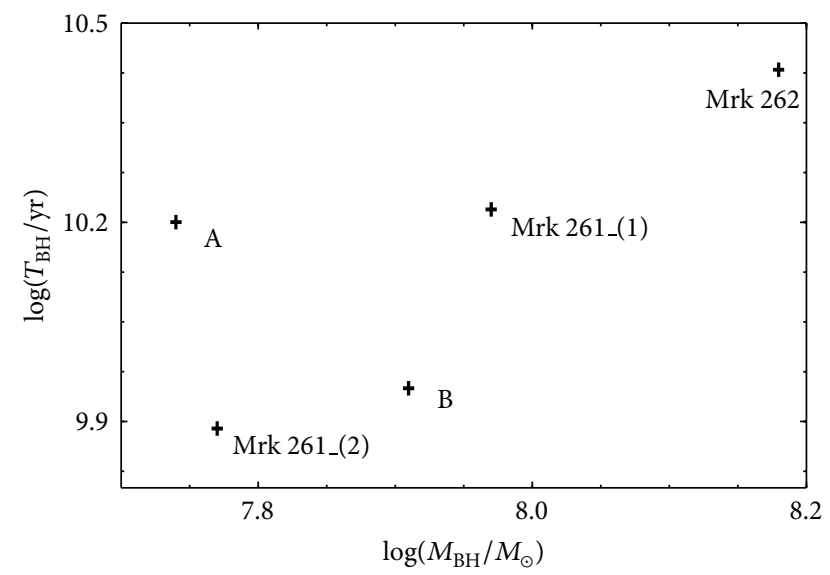

Figure 6: The $M_{\mathrm{BH}} / M_{\odot}$-PRT relation on logarithmic scales in Mrk 261/262 and Mrk 266. The resulting PRTs are of the same scale as the age of the Universe.

Namely, by reiterating integrations of the state equations of SPC-configurations we determine those required central values of particle concentration $n(0)$ and ID-field $x(0)$, for which the total mass of configuration obtained after integration is equal to the black hole mass estimated from observations. Among all the integral characteristics, the radius $R_{d}$ is also computed, which is used to calculate $T_{\mathrm{BH}}$. The results are summed up in Table 3 . The obtained PRT values are ranging from $\log \left(T_{\mathrm{BH}} / \mathrm{yr}\right) \simeq 9.89$ to 10.43 for the masses $\log \left(M_{\mathrm{BH}} / M_{\odot}\right) \simeq 7.77$ to 8.18 of central black holes in 5 nuclear components of the samples of Mrk 261/262 and Mrk 266. Figure 6 plots the PRT-mass relation on logarithmic scales.

\section{UHE Neutrino Fluxes}

We are now in a position to compute the UHE neutrino fluxes. We have chosen the cosmological model favored today, with a flat Universe filled with matter $\Omega_{M}=\rho_{M} / \rho_{c}$ and vacuum
TABLE 3: The PRTs and fluxes for the multinuclei samples Mrk 261/262 and Mrk 266. Columns: (1) name; (2) log of radius (km) of PC; (3) $\log$ of PRT (yr); (4) $J^{q}$ is the log of the $\left(J_{\nu \varepsilon}^{q} / \varepsilon_{d}\right.$ $\left.\operatorname{erg~cm} \mathrm{cm}^{-1} \mathrm{sr}^{-1}\right)$; (5) $J^{\pi}$ is the log of the $\left(J_{\nu \mathcal{E}}^{\pi} / \varepsilon_{d} \operatorname{erg~cm}^{-2} \mathrm{~s}^{-1} \mathrm{sr}^{-1}\right)$; and (6) $J^{u}$ is the log of the $\left(J_{v_{\varepsilon}}^{\mathrm{URCA}} / \varepsilon_{d} \mathrm{erg} \mathrm{cm}^{-2} \mathrm{~s}^{-1} \mathrm{sr}^{-1}\right)$.

\begin{tabular}{lccccc}
\hline Name & $\log \left(R_{d}\right)(\mathrm{km})$ & $\log \left(T_{\mathrm{BH}}\right)(\mathrm{yr})$ & $J_{\varepsilon}^{q}$ & $J_{\varepsilon}^{\pi}$ & $J_{\varepsilon}^{u}$ \\
\hline \multicolumn{5}{c}{ Mrk 261/262 } \\
Mrk261_(1) & -4.13 & 10.22 & -8.55 & -9.51 & -18.69 \\
Mrk261_(2) & -4.0 & 9.89 & -8.22 & -9.17 & -18.35 \\
Mrk 262 & -4.38 & 10.43 & -8.95 & -9.90 & -18.08 \\
\hline \multicolumn{5}{c}{ Mrk 266 } \\
A & -4.07 & 10.20 & -8.09 & -9.04 & -18.22 \\
B & -4.35 & 9.95 & -8.40 & -9.35 & -18.53 \\
\hline
\end{tabular}

energy densities $\Omega_{V}=\rho_{V} / \rho_{c}$. Thereby $\Omega_{V}+\Omega_{M}=1$, where the critical energy density $\rho_{c}=3 H_{0}^{2} /\left(8 \pi G_{N}\right)$ is defined through the Hubble parameter $H_{0}$. For our calculations we have taken the values $H_{0}=70 \mathrm{~km} / \mathrm{s} / \mathrm{Mpc}, \Omega_{V}=0.7$, and $\Omega_{M}=0.3$.

4.1. URCA Reactions. In the framework of MTBH, the total UHE neutrino spectral flux of cooling of the SPC, of given mass $\widetilde{M}$, by modified URCA reactions with no muons, reads (Appendix B.1),

$$
\begin{aligned}
& J_{\nu \varepsilon}^{\mathrm{URCA}} \\
& \simeq 5.22 \times 10^{-8} \frac{\varepsilon_{d}}{I^{2}(z)(1+z)}\left(\frac{M_{\odot}}{\widetilde{M}}\right)^{1.75} \mathrm{erg} \mathrm{cm}^{-2} \mathrm{~s}^{-1} \mathrm{sr}^{-1},
\end{aligned}
$$

where the neutrino is radiated in a cone with the beaming angle $\theta \sim \varepsilon_{d}=d / 2 R_{g}, d$ is the thickness of the protomatter disk at the edge of even horizon and $I(z)=(1+$ z) $\int_{1}^{1+z} d x / \sqrt{2.3+x^{3}}$. As it is seen, the nucleon modified URCA reactions can contribute efficiently only for extragalactic objects with enough small redshift $z \ll 1$.

4.2. Pionic Reactions. The pionic reactions, which occurred in the superdense protomatter medium of SPC, allow both the distorted energy and momentum to be conserved. The following is the analogue of the simple URCA processes:

$$
\pi^{-}+n \longrightarrow n+e^{-}+\bar{v}_{e}, \quad \pi^{-}+n \longrightarrow n+\mu^{-}+\bar{v}_{\mu},
$$

and the two inverse processes. As in the modified URCA reactions, the total rate for all four processes is essentially four times the rate of each reaction alone. The muons are already present when pions appear. The UHE neutrino mean flux is given (Appendix B.2) as

$$
J_{\nu \varepsilon}^{\pi} \simeq 7.91 \frac{\varepsilon_{d}}{I^{2}(z)(1+z)}\left(\frac{M_{\odot}}{\widetilde{M}}\right)^{1.75} \operatorname{erg~cm}^{-2} \mathrm{~s}^{-1} \mathrm{sr}^{-1} .
$$

The resulting total energy-loss rate will then be dramatically larger due to the pionic reactions (5) rather than the modified URCA processes. 
4.3. Quark Reactions. In the superdense protomatter medium, the distorted quark Fermi energies are far below the charmed $c_{-}, t-$, and $b$-quark production thresholds. Therefore, only up, down, and strange quarks are present. The $\beta$ equilibrium is maintained by reactions like

$$
\begin{array}{ll}
d \longrightarrow u+e^{-}+\bar{v}_{e}, & u+e^{-} \longrightarrow d+v_{e}, \\
s \longrightarrow u+e^{-}+\bar{v}_{e}, & u+e^{-} \longrightarrow s+v_{e},
\end{array}
$$

which are $\beta$ decay and its inverse. These reactions constitute simple URCA processes, in which there is a net loss of a $\nu_{l} \bar{\nu}_{l}$ pair at nonzero temperatures. In this application, a sufficient accuracy is obtained by assuming $\beta$ equilibrium and that the neutrinos not to be retained in the medium of $\Lambda$-like protomatter. The quark reactions (7) and (8) proceed at equal rates in $\beta$ equilibrium, where the participating quarks must reside close to their Fermi surface. Hence, the total energy of flux due to simple URCA processes is rather twice that of (7) or (8) alone. The total flux of UHE neutrino can be written (Appendix B.3) as

$$
J_{\nu \mathcal{E}}^{q} \simeq 70.68 \frac{\varepsilon_{d}}{I^{2}(z)(1+z)}\left(\frac{M_{\odot}}{\widetilde{M}}\right)^{1.75} \mathrm{erg} \mathrm{cm}^{-2} \mathrm{~s}^{-1} \mathrm{sr}^{-1} .
$$

4.4. Total Flux Simulation. For total flux simulation we use the data of all the black hole mass and luminosity estimates from Table 2. Solving the inverse problem, we determine the radii and surface temperatures corresponding to these sources. The results of the computation are summed up in the Table 3, and are shown in Figures 7, 8, and 9. The last three columns present the neutrino fluxes $F_{\varepsilon}^{i}(i=q, \pi, u)$, which are

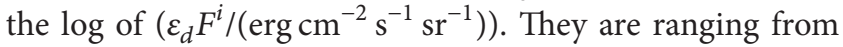
$J_{\nu \varepsilon}^{q} /\left(\varepsilon_{d} \operatorname{erg~cm}^{-2} \mathrm{~s}^{-1} \mathrm{sr}^{-1}\right) \simeq 1.1 \times 10^{-9}$ to $8.2 \times 10^{-9} ; J_{\nu \varepsilon}^{\pi} \simeq$ $0.1 J_{\nu \varepsilon}^{q} ; J_{\nu \mathcal{E}}^{\mathrm{URCA}} \simeq 7.4 \times 10^{-11} J_{\nu \mathcal{\varepsilon}}^{q}$.

\section{Conclusions}

In the framework of $\mathrm{MTBH}$, we address the neutrino radiation of plausible accreting supermassive black holes closely linked to 5 nuclear components of unusual galaxy samples of Mrk261/262 and Mrk 266. This necessarily requires to estimate more accurately the values of black hole masses and bolometric luminosities for these nuclear components. We estimate these values from our observations and predict an 10 Gyr time delay before neutrino emission. The UHE neutrinos with energies up to hundreds $\mathrm{ZeV}$ are produced in superdense protomatter medium via simple (quark and pionic reactions) or modified URCA processes, even after the neutrino trapping. The computed neutrino fluxes for quark reactions are ranging from $J_{\nu \varepsilon}^{q} /\left(\varepsilon_{d} \operatorname{erg~cm}^{-2} \mathrm{~s}^{-1} \mathrm{sr}^{-1}\right) \simeq$ $1.1 \times 10^{-9}$ to $8.2 \times 10^{-9}$. For pionic and modified URCA reactions, the fluxes are $J_{\nu \mathcal{E}}^{\pi} \simeq 0.1 J_{\nu \mathcal{E}}^{q}$ and $J_{\nu \mathcal{E}}^{\mathrm{URCA}} \simeq 7.4 \times$ $10^{-11} J_{\nu \mathcal{E}}^{q}$, respectively. These fluxes are highly beamed along the plane of accretion disk, peaked at ultrahigh energies and collimated in smaller opening angle $\varepsilon_{d} \sim \theta \sim 10^{-12}$. The Mrk 261/262 and Mrk 266, therefore, may be of interest for the Z-burst scenario of origin of UHECRs. Such neutrinos may

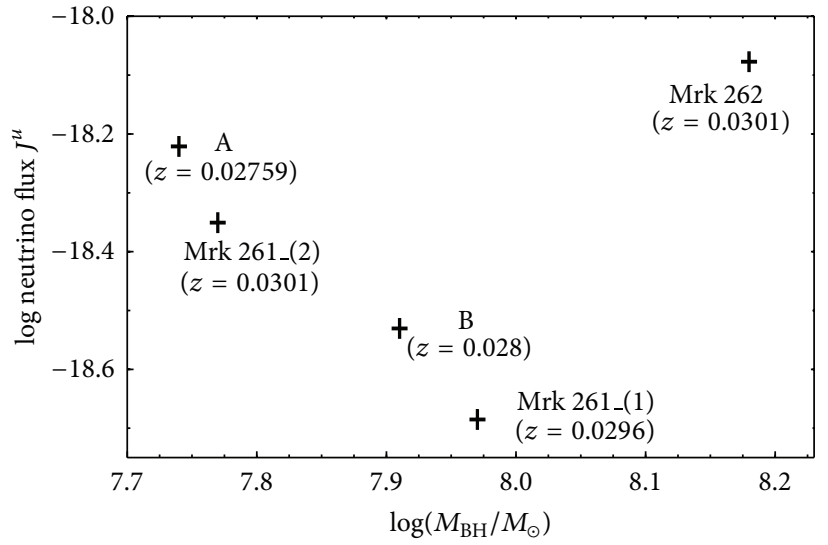

FIGURE 7: UHE neutrino fluxes on logarithmic scales of the plausible black holes in 5 nuclear components in Mrk 261/262 and Mrk 266. $J^{u}$ is the $\log$ of $\left(J_{v \varepsilon}^{\mathrm{URCA}} / \varepsilon_{d} \operatorname{erg~cm}^{-2} \mathrm{~s}^{-1} \mathrm{sr}^{-1}\right)$.

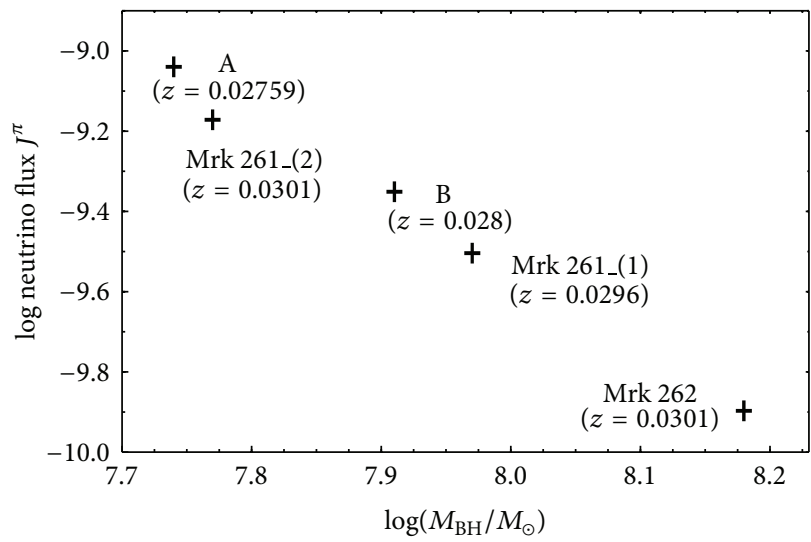

FIGURE 8: UHE neutrino fluxes on logarithmic scales of the plausible black holes in 5 nuclear components in Mrk 261/262 and Mrk 266.

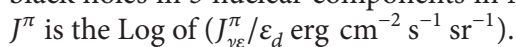

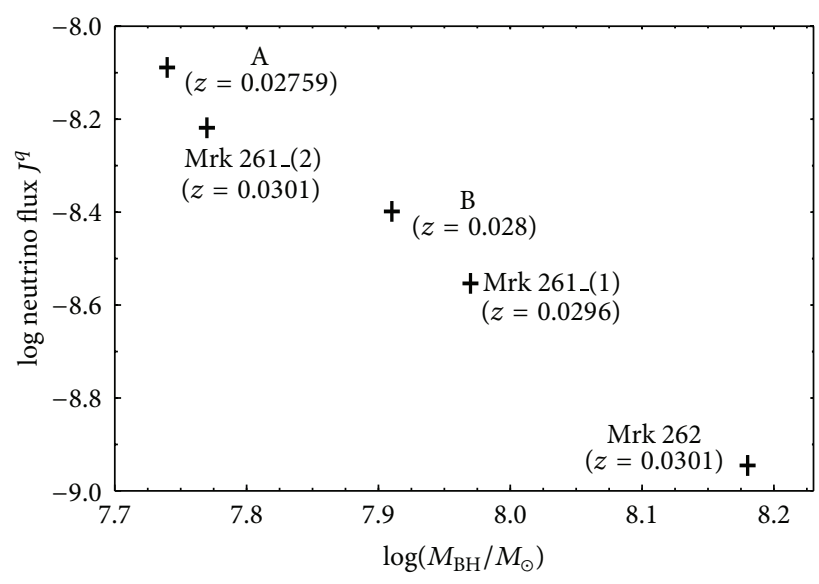

FIGURE 9: UHE neutrino fluxes on logarithmic scales of the plausible black holes in 5 nuclear components in Mrk 261/262 and Mrk 266. $J^{q}$ is the $\log$ of $\left(J_{\gamma \varepsilon}^{q} / \varepsilon_{d} \operatorname{erg~cm}^{-2} \mathrm{~s}^{-1} \mathrm{sr}^{-1}\right)$. 


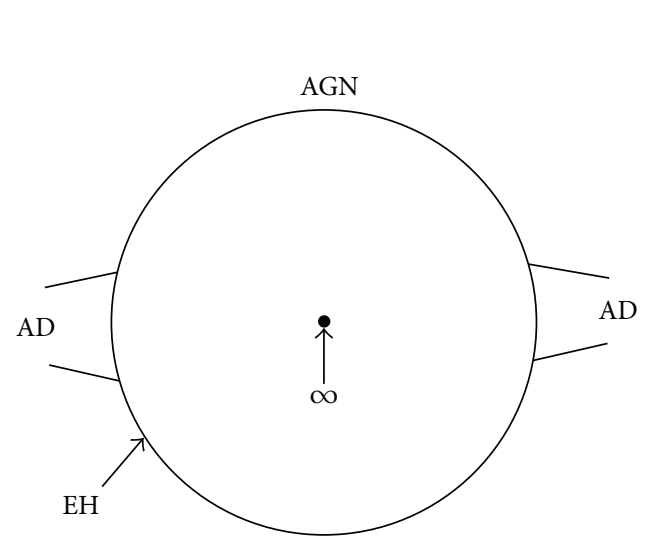

(a)

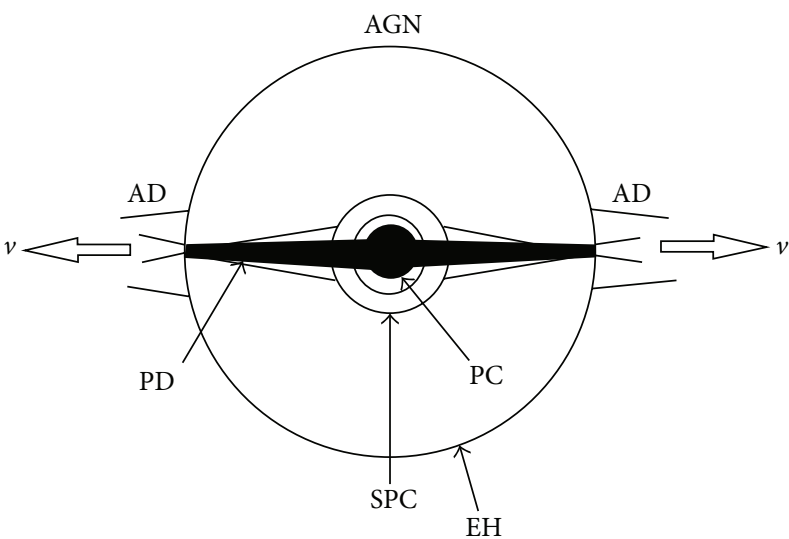

(b)

FIGURE 10: EH: event horizon, AD: accretion disk, SPC: superdense protomatter core, and PC: protomatter core. (a) The phenomenological model of AGN with the central stationary black hole. The meaningless singularity occurs at the center inside of the black hole. Either the Kruskal continuation of the metric for a Schwarzschild black hole or the Kerr metric shows that the static observers do not exist inside the horizon. Any timelike world line inside the EH must strike the central singularity which wholly absorbs the infalling particles. This disables an accumulation of matter in the central part, and, thus, neither a growth of black hole nor an increase of its mass-energy density occur at accretion of outside matter. (b) The microscopic model of AGN with central stable SPC, due to which the stable equilibrium holds in outward layers too and, thus, now an accumulation of matter allowed around SPC inside the EH. The static observers can exist inside the EH. The SPC accommodates the highest energy scale $>10^{21} \mathrm{eV}$ in PC. With time, an infalling matter forms PD around PC tapering off faster at reaching out the thin edge of EH. At this, metric singularity cutoff $[6,7]$ is switched on and the neutrinos of huge energies may escape through the vista to the outside world.

initiate the cascades of UHE cosmic rays via very complex chains of Z-burst interactions. Some part of UHE neutrinos may produce in accretion disk and in a torus of hot gas surrounding the AGN core the secondary electrons with huge energies. The latter, in turn, may give rise to a secondary flux of the $\mathrm{GeV}-\mathrm{TeV}$ gamma rays.

\section{Appendices}

\section{A. Further Topics on the MTBH}

In this section we recollect some relevant points of the $\mathrm{MTBH}$ $[6,7]$ in a concise schematic form.

A.1. The Stable SPC. Discussed in $[6,15,16]$ gravitational theory is consistent with general relativity up to the limit of neutron star. However, due to the spontaneous gauge symmetry breaking in gravity, it also manifests its virtues in density range far above nuclear density. The black hole model, presented in the framework of phenomenological and microscopic approaches, is schematically plotted in Figure 10. The equations describing the equilibrium SPC include the gravitational and ID field equations, the hydrostatic equilibrium equation, and the state equation. The latter is specified for each domain of many layered configurations. Each particle of the protomatter goes off from its original mass shell and, thus, acquires the distorted energy, momentum and mass at rest. The thermodynamics of the protomatter strongly differs from the thermodynamics of highly compressed ordinary matter.
The resulting stable SPC is formed, which consists of the protomatter core and the outer layers of ordinary matter.

SPC-Configurations in More Details. A layering of configurations is a consequence of the onset of different regimes in equation of state. In the density range $\rho<4.54 \times 10^{12} \mathrm{~g} \mathrm{~cm}^{-3}$, one uses for both configurations the simple semiempirical formula of state equation given by [49]. Above the density $\rho>4.54 \times 10^{12} \mathrm{~g} \mathrm{~cm}^{-3}$, for the simplicity, the I-class $\mathrm{SPC}_{\mathrm{I}}$ configuration is thought to be composed of regular neutronproton-electron (n-p-e) gas (in absence of ID) in intermediate density domain $4.54 \times 10^{12} \mathrm{~g} \mathrm{~cm}^{-3} \leq \rho<\rho_{d}$ and of the n-p-e protomatter in presence of ID at $\rho>\rho_{d}$. For the II-class SPC $\mathrm{II}$ configuration above the density $\rho_{f l}=4.09 \times$ $10^{14} \mathrm{~g} \mathrm{~cm}^{-3}$, one considers an onset of melting down of hadrons when nuclear matter consequently turns to quark matter, found in string flip-flop regime. In domain $\rho_{f l} \leq$ $\rho<\rho_{d}$, to which the distances $0.4 \mathrm{fm}<r_{\mathrm{NN}} \leq 1.6 \mathrm{fm}$ correspond, one has the regular (ID is absent) string flipflop regime. This is a kind of tunneling effect when the strings joining the quarks stretch themselves violating energy conservation and after touching each other they switch on to the other configuration [6]. In domain $\rho_{d} \leq \rho<\rho_{a s}$, one has the string flip-flop regime in presence of ID, at distances $0.25 \mathrm{fm}<r_{\mathrm{NN}} \leq 0.4 \mathrm{fm}$. That is, the system is made of quark protomatter in complete $\beta$ equilibrium with rearrangement of string connections joining them. In the final domain $\rho>\rho_{a s}$, the system is made of quarks in one bag in complete $\beta$ equilibrium at presence of ID. The quarks are under the weak interactions and gluons, including 
the effects of QCD-perturbative interactions. The QCD vacuum has a complicated structure, which is intimately connected to the gluon-gluon interaction. In most applications, sufficient accuracy is obtained by assuming that all quarks are almost massless inside a bag. This physical picture with appropriate modifications is adapted by [6] to the medium of quark protomatter. The latter is regarded as a noninteracting Fermi gas found in the ID region of the space-time continuum, at short distances $r_{\mathrm{NN}} \leq 0.25 \mathrm{fm}$. Each configuration is defined by the two free parameters of central values of particle concentration $\widetilde{n}(0)$ and dimensionless potential of space-like ID-field $x(0)$. The interior gravitational potential $x_{0}^{\text {int }}(r)$ matches into the exterior one $x_{0}^{\text {ext }}(r)$ at the surface of the configuration. The central value of the gravitational potential $x_{0}(0)$ can be found by reiterating integrations when the sewing condition of interior and exterior potentials holds. The simulations confirm in brief the following scenario: the energy density and internal pressure have sharply increased in protomatter core (with respect to corresponding central values of neutron star) proportional to gravitational forces of compression. This counteracts the collapse, and equilibrium holds even for the masses $\sim 10^{9} M_{\odot}$. The most important question of stability of SPC was further studied in [7], whereas minimizing the energy $\widetilde{E}$ gives the equilibrium configuration. The second derivative of $\widetilde{E}$ gives stability information. A relativity tends to destabilize configurations. However, a numerical integration of the stability equations of SPC clearly proves the stability of resulting SPC. Note that, since SPC is always found inside the event horizon sphere, then it could be observed only in presence of accreting matter.

A.2. PRT versus Bolometric Luminosity. In approximation $R_{d} \ll R_{g}$, the PRT reads

$$
T_{\mathrm{BH}}=\rho_{d} \frac{V_{d}}{\dot{M}} \simeq 9.33 \cdot 10^{15} \mathrm{~g} \mathrm{~cm}^{-3} \frac{R_{d} R_{g}^{2}}{\dot{M}} .
$$

In case of collisionless accretion, (A.1) gives $T_{\mathrm{BH}}[7]$ as follows:

$$
T_{\mathrm{BH}} \simeq 2.6 \cdot 10^{16} \frac{R_{d}}{\mathrm{~cm}} \frac{10^{-24} \mathrm{~g} \mathrm{~cm}^{-3}}{\rho_{\infty}} \frac{v_{\infty}}{10 \mathrm{~km} \mathrm{~s}^{-1}} \mathrm{yr} .
$$

For the hydrodynamic accretion [7], we get

$$
T_{\mathrm{BH}} \simeq 8.8 \cdot 10^{38} \frac{R_{d} R_{g}^{2} \mathrm{~cm}^{-3}}{n_{s} r_{s}^{5 / 2}\left(\ln g_{00}\right)_{s}^{\prime}},
$$

where $\left(g_{00}\right)_{s} \equiv g_{00}\left(r_{s}\right), r_{s}$ is the critical sonic point outside the event horizon. The spherical accretion onto black hole, in general, is not necessarily an efficient mechanism for converting rest-mass energy into radiation. Accretion onto black hole may be far from spherical accretion, because the accreted gas possesses angular momentum. In this case, the gas will be thrown into circular orbits about the black hole when centrifugal forces will become significant before the gas plunges through the event horizon [7]. According to (A.2) and (A.3), the resulting relationship of typical PRT versus bolometric luminosity becomes

$$
T_{\mathrm{BH}} \simeq 0.17 \frac{R_{d}}{R_{a}}\left(\frac{M_{\mathrm{BH}}}{M_{\odot}}\right)^{3} \frac{10^{39} W}{L_{\mathrm{bol}}} \mathrm{yr} .
$$

Assuming a typical mass-energy conversion efficiency of about $\epsilon \sim 10 \%$, we recast this equation into the final form of (3).

\section{B. UHE Neutrino Fluxes}

Hereafter, the following notational conventions will be used: we denote the quantities referred to SPC by wiggles, while the corresponding quantities of neutron star or quark star are left without wiggles. The interior temperature or temperature at the surface of protomatter core is $\widetilde{T}>100 \mathrm{ZeV} / k_{B} \sim 1.2 \times$ $10^{27} \mathrm{~K}[6]$. The SPC interiors are to a good approximation isothermal, but near the surface the temperature $\widetilde{T}_{e}$ drops rapidly. We may, therefore, adapt a standard discussion of the degenerate-nondegenerate transition region of a neutron star to the corresponding region of SPC. This is quick to estimate $\widetilde{T}_{e}$ and will guide us toward a first look at some of the associated physics. So, the surface temperature $\widetilde{T}_{e}$ of the SPC can be given [6] as follows:

$$
\frac{\widetilde{T}_{e}}{T_{e}} \simeq 3.5 \times 10^{7}\left(\frac{\widetilde{M}}{M_{\odot}}\right)^{-1 / 4} .
$$

B.1. URCA Reactions. Given a luminosity of the neutron star of mass $M$ and a uniform density $\rho$ by modified URCA reactions with no muons $[50]$

$$
L_{v}^{\mathrm{URCA}} \simeq 5.3 \times 10^{39} \frac{M}{M_{\odot}}\left(\frac{\rho_{\mathrm{nuc}}}{\rho}\right)^{1 / 3} T_{9}^{8} \mathrm{erg} \mathrm{s}^{-1},
$$

it is straightforward to estimate a luminosity of SPC, because all of 8 powers of the temperature arisen in (B.2) are in the phase-space factor. Here $\rho_{\text {nuc }}=2.8 \times 10^{14} \mathrm{~g} \mathrm{~cm}^{-3}$. Such approach is much less time-consuming than a direct calculation. We therefore expect a resulting luminosity of SPC of a density $\widetilde{\rho} \sim \widetilde{p}_{F}(n) \widetilde{p}_{F}(p) \widetilde{p}_{F}(e)$ to be

$$
\widetilde{L}_{\nu \mathcal{E}}^{\mathrm{URCA}}=\varepsilon \frac{\widetilde{M}}{M_{\odot}}\left(\frac{\widetilde{\rho}}{\rho}\right)^{4 / 3}\left(\frac{\widetilde{T}_{e}}{T_{e}}\right)^{3} L_{\nu}^{\mathrm{URCA}} .
$$

The coefficient $\varepsilon$ reads

$$
\varepsilon=\varepsilon_{d} \varepsilon_{\text {trap }},
$$

where $\varepsilon_{\text {trap }}$ is the neutrino trapping coefficient, which is arisen as the neutrinos that are trapped comove with matter and build up a semidegenerate Fermi sea [6] as follows:

$$
\begin{aligned}
\varepsilon_{\text {trap }} & \simeq 1.1 \times 10^{-5}\left(\frac{12 \mathrm{~km}}{\widetilde{R}}\right)^{2}\left(\frac{\rho_{\text {nuc }}}{\widetilde{\rho_{d}}}\right)^{13 / 6} \\
& \simeq 1.54 \times 10^{-7}\left(\frac{M_{\odot}}{\widetilde{M}}\right)^{2} .
\end{aligned}
$$


We obtain then

$$
\widetilde{L}_{\nu \varepsilon}^{\mathrm{URCA}}=3.8 \times 10^{50} \varepsilon_{d}\left(\frac{M_{\odot}}{\widetilde{M}}\right)^{1.75} \mathrm{erg} \mathrm{s}^{-1} .
$$

Consequently, the flux reads

$$
J_{\nu \varepsilon}^{\mathrm{URCA}}=\frac{\widetilde{L}_{v \varepsilon}^{\mathrm{URCA}}}{4 \pi D_{L}^{2}(z)(1+z)}
$$

where $z$ is the redshift and $D_{L}(z)$ is the luminosity distance depending on the cosmological model. The $(1+z)^{-1}$ is due to the fact that each neutrino with energy $\widetilde{E}_{v}^{\prime}$ if observed near the place and time of emission $t^{\prime}$ will be red-shifted to energy $\widetilde{E}_{\gamma}=\widetilde{E}_{\gamma}^{\prime} R\left(t_{1}\right) / R\left(t_{0}\right)=\widetilde{E}_{\gamma}^{\prime}(1+z)^{-1}$ of the neutrino observed at time $t$ after its long journey to us, where $R(t)$ is the cosmic scale factor. The luminosity distance is given as [26]

$$
\begin{aligned}
D_{L}(z) & =\frac{(1+z) c}{H_{0} \sqrt{\Omega_{M}}} \int_{1}^{1+z} \frac{d x}{\sqrt{\left(\Omega_{V} / \Omega_{M}\right)+x^{3}}} \\
& =2.4 \times 10^{28} I(z) \mathrm{cm},
\end{aligned}
$$

where $I(z)=(1+z) \int_{1}^{1+z} d x / \sqrt{2.3+x^{3}}$, and we have taken the values $H_{0}=70 \mathrm{~km} / \mathrm{s} / \mathrm{Mpc}, \Omega_{V}=0.7$, and $\Omega_{M}=0.3$. Combining (B.6)-(B.8), we obtain the total flux (4).

B.2. Pionic Reactions. Note that there are two fewer fermions participating in the reactions than in the modified URCA reactions. So the phase-space factor varies as $T^{6}$ rather than $T^{8}$. Continuing along the previous section, we obtain then

$$
\widetilde{L}_{\nu \varepsilon}^{\pi}=\varepsilon \frac{\widetilde{M}}{M} \frac{\rho}{\tilde{\rho}}\left(\frac{\widetilde{T}_{F}}{T_{F}}\right)^{3}\left(\frac{\widetilde{T}_{e}}{T_{e}}\right)^{3} L_{\nu}^{\pi},
$$

where $\widetilde{T}_{F}=\widetilde{E}_{F} / k_{B}, L_{v}^{\pi}$ refers to the neutron star [51] as follows:

$$
L_{v}^{\pi} \simeq 1.5 \times 10^{46} \vartheta^{2} \frac{M}{M_{\odot}} \frac{\rho_{\text {nuc }}}{\rho} T_{9}^{6} \operatorname{erg~s}^{-1},
$$

and $\vartheta \sim 0.3$ is an angle measuring the degree of pion condensation. Hence,

$$
\widetilde{L}_{\nu \varepsilon}^{\pi}=5.78 \times 10^{58} \varepsilon_{d}\left(\frac{M_{\odot}}{\widetilde{M}}\right)^{1.75} \mathrm{erg} \mathrm{s}^{-1}
$$

and the total UHE neutrino flux is then given by (6).

B.3. Quark Reactions. Our purpose here is in sufficient approximation to adapt the discussion of ordinary quark matter in neutron star $[52,53]$ to the corresponding region of quark protomatter in SPC. A rearrangement of vacuum state causes a shift of zero point energy of the protomatter particles. Accordingly, the energy-loss rate of the SPC due to neutrino emission process $d \rightarrow u+e^{-}+\bar{v}_{e}$ is generalized in the following form [6]:

$$
\begin{aligned}
\widetilde{\epsilon}_{\bar{\nu} \varepsilon}^{q}= & 6 V^{-1} \varepsilon\left(\prod_{i=1}^{4} V \int \frac{d^{3} \widetilde{p}_{i}}{(2 \pi)^{3}}\right) \widetilde{E}_{\bar{\nu}} V(2 \pi)^{4} \delta^{(4)} \\
& \times\left(\widetilde{p}_{d}-\widetilde{p}_{\bar{\nu}_{e}}-\widetilde{p}_{u}-\widetilde{p}_{e}\right) \frac{|M|^{2}}{\prod_{i=1}^{4} 2 \widetilde{E}_{i} V} S,
\end{aligned}
$$

where $\widetilde{p}_{i}$ and $\widetilde{E}_{i}$ denote the distorted momentum and energy of a particle of the given species of protomatter. The four vectors $\widetilde{p}_{i}$ are numbered as $i=1,2,3,4 \equiv d, \bar{v}_{e}, u, e^{-}, V$ is the normalization volume, $|M|^{2}$ is the squared invariant amplitude averaged over the initial $d$-quark spin and summed over the final spins of the $u$ quark and the electron, $f \equiv$ $\left[\exp \widetilde{\beta}\left(\widetilde{E}_{i}-\widetilde{\mu}_{i}\right)+1\right]^{-1}$ is the fraction of phase space occupied at energy $\widetilde{E}_{i}$ and distorted chemical potential $\widetilde{\mu}_{i}=(1+$ $\left.8 \alpha_{c} / 3 \pi\right) c \widetilde{p}_{F i}$, given to the lowest order in the QCD interaction coupling constant $\alpha_{c}, \widetilde{p}_{F i}$ is the Fermi momentum, and $S=$ $f_{d}\left(1-f_{u}\right)\left(1-f_{e}\right)$ is the statistical factor. The blocking factors $1-f_{i}$ accounting for the distribution of final states reduce the reaction rate, which ensure that the exclusion principle is obeyed; the factor 3 takes account of three color degrees of freedom, and 2 is the spin of the initial $d$ quark. Since typical Fermi energies of quarks are higher than the electron rest mass, a slight difference between $\tilde{\mu}_{d}$ and $\tilde{\mu}_{e}$ (or $\tilde{\mu}_{s}$ and $\tilde{\mu}_{u}$ ) implies that the electrons will generally have a relativistic Fermi energy. The interaction of Lagrangian density is given in the standard current-current form. As the neutrinos are produced thermally, we can neglect the neutrino momentum in the momentum conservation law. We may further set $\widetilde{E}_{i} \simeq \widetilde{p}_{i}$ because all the particles are relativistic and that $\tilde{p}_{d} \sim \tilde{p}_{u}\left(\tilde{p}_{e} \ll \tilde{p}_{d}\right)$. Also all the $\tilde{p}_{i}$ (except $\left.\tilde{p}_{\bar{\gamma}}\right)$ can be set equal $\widetilde{p}_{F}(i)$ and removed from the integral. To estimate the magnitude of the emissivity (B.12), we follow the original argument presented in [52], which applies to both neutron star and SPC, and assume that for distorted Fermi momenta $\widetilde{p}_{F}(d) \simeq \widetilde{p}_{F}(u) \simeq \widetilde{p}_{F}(s)$ as in noninteracting case, such that $\widetilde{p}_{F}(e) \simeq 3^{(1 / 3)} \widetilde{Y}_{e}^{(1 / 3)} \widetilde{p}_{F}(q)$, where $\widetilde{Y}_{e} \simeq \widetilde{n}_{e} / \widetilde{n}_{b}, \widetilde{n}_{i}$ is the distorted concentration of particles. We also set the value $\alpha_{c} \simeq 0.1$ as rather being a reasonable order of magnitude estimate and $\widetilde{Y}_{e} \simeq 0.01$ as typical value of what is expected for superdense protomatter. The statistical factor is $S=f_{d}\left(1-f_{u}\right)\left(1-f_{e}\right)=$ $\prod_{j=1}^{3}\left(1+e^{x_{j}}\right)^{-1}$, where the dimensionless energies $x_{j}$ are defined $x_{1}=\widetilde{\beta}\left(\widetilde{E}_{d}-\widetilde{\mu}_{d}\right), x_{2}=-\widetilde{\beta}\left(\widetilde{E}_{u}-\widetilde{\mu}_{u}\right), x_{1}=-\widetilde{\beta}\left(\widetilde{E}_{e}-\widetilde{\mu}_{e}\right)$. There will therefore be [6] as follows:

$$
\widetilde{\epsilon}_{\overline{\nu \varepsilon}}^{q}=A \tilde{p}_{F}(d) \tilde{p}_{F}(u) \tilde{p}_{F}(e) \tilde{\beta}^{-6} I,
$$

provided,

$$
\begin{gathered}
I=\int_{0}^{\infty} d y_{2} y_{2}^{3} \mathcal{J}, \\
\mathscr{J}=\int \sum_{j=1}^{3}\left(1+e^{x_{j}}\right)^{-1} \delta\left[\sum_{j=1}^{3} x_{j}-y_{2}\right],
\end{gathered}
$$


where $A=16 \pi^{-6} \varepsilon \alpha_{c} G^{2} \cos ^{2} \theta_{C}, y_{2} \equiv \widetilde{E}_{\bar{\nu}} \widetilde{\beta}$, the weak-coupling constant is $G \simeq 1.435 \times 10^{-40} \mathrm{erg} \mathrm{cm}^{3}$, and $\theta_{C}$ is the Cabibbo angle ( $\left.\cos ^{2} \theta_{C} \simeq 0.948\right)$. Recall that a standard evaluation of $\mathscr{F}$ in the complex plane gives [49]

$$
\left(1+e^{y_{2}}\right) \mathscr{J}=\text { Residue at } z=0 \text { of }\left[e^{-i z y_{2}}\left(\frac{\pi}{\sinh \pi z}\right)^{3}\right]
$$

where residue is found by making series expansions of the exponential and the sinh about $z=0$ and reading off the coefficient $1 / 2$ such that

$$
\left(1+e^{y_{2}}\right) \mathscr{J}=\frac{1}{2}\left(\pi^{2}+y_{2}^{2}\right) .
$$

Taking into account that for each degenerate species only a fraction $\widetilde{T} / \widetilde{T}_{F}$ effectively contributes to the cooling rate and that there are one such initial species and two such final species, finally we arrive at the spectral flux of the UHE antineutrinos:

$$
\begin{aligned}
& \frac{d J_{\bar{\nu} \varepsilon}^{q}}{d y_{2}} \\
& \simeq 0.41 \frac{\varepsilon_{d}}{I^{2}(z)(1+z)}\left(\frac{M_{\odot}}{\widetilde{M}}\right)^{1.75} \frac{y_{2}^{3}\left(\pi^{2}+y_{2}^{2}\right)}{e^{y_{2}}+1} \mathrm{erg} \mathrm{cm}^{-2} \mathrm{~s}^{-1} \mathrm{sr}^{-1} .
\end{aligned}
$$

Similar reasoning can be applied for the process $u+e^{-} \rightarrow$ $d+v_{e}$. We obtain the resulting spectral UHE neutrino flux

$$
\begin{aligned}
& \frac{d J_{\nu \varepsilon}^{q}}{d y_{1}} \\
& \simeq 0.1 \frac{\varepsilon_{d}}{I^{2}(z)(1+z)}\left(\frac{M_{\odot}}{\widetilde{M}}\right)^{1.75} \frac{y_{1}^{4}\left(\pi^{2}+y_{1}^{2}\right)}{e^{y_{1}}+1} \mathrm{erg} \mathrm{cm}^{-2} \mathrm{~s}^{-1} \mathrm{sr}^{-1} .
\end{aligned}
$$

Different $\widetilde{E}_{v}$ dependence is due to the partial restriction of the electron's phase space which introduces an extra factor of $\left(\widetilde{E}_{e} \widetilde{\beta}\right)^{-1}$. But, as far as (B.18) is independent of the electron's Fermi energy, a potential source of ambiguity is eliminated. Consequently, both (B.17) and (B.18) yield the total flux (9).

\section{Acknowledgments}

The authors appreciate the very helpful and knowledgeable comments from the anonymous referees that have essentially clarified the paper.

\section{References}

[1] R. C. Kennicutt Jr., "Star formation in galaxies along the Hubble sequence," Annual Review of Astronomy and Astrophysics, vol. 36, no. 1, pp. 189-231, 1998.

[2] V. A. Ambartsumian, "Some remarks on multiple galaxies," in Proceedings of the 5th IAU Symposium, N. Grace Roman, Ed., p. 4, Cambridge University Press, Dublin, Ireland, September 1955.
[3] V. A. Ambartsumian, "Instability phenomena in systems of galaxies," Astronomical Journal, vol. 66, pp. 536-540, 1961.

[4] V. A. Ambartsumian, "The nuclei of galaxies and their activity," in The Structure and Evolution of Galaxies, Library of Congress Catalog Card No. 65-26979, p. 1, Interscience Publishers, A Division of John Wiley \& Sons, London, UK, 1965.

[5] E. Khachikian, G. Ter-Kazarian, L. Sargsyan, K. Yerknapetyan, and Y. Terzian, "On multi-nuclei features of some Markarian galaxies," Monthly Notices of the Royal Astronomical Society, vol. 368, no. 1, pp. 461-470, 2006.

[6] G. T. Ter-Kazarian, "Protomatter and EHE C.R," Journal of the Physical Society of Japan B, vol. 70, pp. 84-98, 2001.

[7] G. Ter-Kazarian, S. Shidhani, and L. Sargsyan, "Neutrino radiation of the AGN black holes," Astrophysics and Space Science, vol. 310, no. 1-2, pp. 93-110, 2007.

[8] P. Natarajan and E. Treister, "Angular momentum and clustering properties of early dark matter haloes," Monthly Notices of the Royal Astronomical Society, vol. 393, pp. 838-845, 2009.

[9] M. Vestergaard, "Early growth and efficient accretion of massive black holes at high redshift," The Astrophysical Journal, vol. 601, no. 2, pp. 676-691, 2004.

[10] M. Volonteri, G. Lodato, and P. Natarajan, "The evolution of massive black hole seeds," Monthly Notices of the Royal Astronomical Society, vol. 383, no. 3, pp. 1079-1088, 2008.

[11] M. Volonteri and P. Natarajan, "Journey to the $M_{\mathrm{BH}}-\sigma$ relation: the fate of low-mass black holes in the universe," Monthly Notices of the Royal Astronomical Society, vol. 400, no. 4, pp. 1911-1918, 2009.

[12] F. Shankar, D. H. Weinberg, and J. Miralda-Escudé, "Selfconsistent models of the AGN and black hole populations: duty cycles, accretion rates, and the mean radiative efficiency," The Astrophysical Journal, vol. 690, no. 1, pp. 20-41, 2009.

[13] B. C. Kelly, M. Vestergaard, X. Fan, P. Hopkins, L. Hernquist, and A. Siemiginowska, "Constraints on black hole growth, Quasar lifetimes, and Eddington ratio distributions from the SDSS broad-line Quasar black hole mass function," The Astrophysical Journal, vol. 719, no. 2, pp. 1315-1334, 2010.

[14] E. Treister and C. M. Urry, "The cosmic history of black hole growth from deep multiwavelength surveys," Advances in Astronomy, vol. 2012, Article ID 516193, 21 pages, 2012.

[15] G. T. Ter-Kazarian, "Gravitation gauge group," Nuovo Cimento della Societa Italiana di Fisica B, vol. 112, no. 6, pp. 825-838, 1997.

[16] G. T. Ter-Kazarian, "Gravitation and inertia; a rearrangement of vacuum in gravity," Astrophysics and Space Science, vol. 327, no. 1, pp. 91-109, 2010.

[17] J.-H. Woo and C. M. Urry, "Active galactic nucleus black hole masses and bolometric luminosities," The Astrophysical Journal, vol. 579, no. 2, pp. 530-544, 2002.

[18] T. J. Weiler, "Resonant absorption of cosmic-ray neutrinos by the relic-neutrinobackground," Physical Review Letters, vol. 49, no. 3, pp. 234-237, 1982.

[19] T. J. Weiler, "Big bang cosmology, relic neutrinos, and absorption of neutrino cosmic rays," The Astrophysical Journal, vol. 285, pp. 495-500, 1984.

[20] T. J. Weiler, "Cosmic-ray neutrino annihilation on relic neutrinos revisited: a mechanism for generating air showers above the Greisen-Zatsepin-Kuzmin cutoff," Astroparticle Physics, vol. 11, no. 3, pp. 303-316, 1999.

[21] D. Fargion, B. Mele, and A. Salis, "Ultra-high-energy neutrino scattering onto relic light neutrinos in the galactic halo as a possible source of the highest energy extragalactic cosmic rays," 
The Astrophysical Journal Letters, vol. 517, no. 2, pp. 725-733, 1999.

[22] D. Fargion, V. K. Dubrovich, and M. Y. Khlopov, "Primordial bound systems of superheavy particles as the source of ultrahigh energy cosmic rays," Nuclear Physics B, vol. 136, no. 1-3, pp. 362-367, 2004.

[23] A. Datta, D. Fargion, and B. Mele, "Supersymmetry-neutrino unbound," Journal of High Energy Physics, vol. 509, article 7, 2005.

[24] P. Jain and S. Panda, "Ultra high energy cosmic rays from early decaying primordial black holes," in Proceedings of the 29th International Cosmic Ray Conference, B. Sripathi Acharya, S. Gupta, P. Jagadeesan et al., Eds., vol. 9, p. 33, Tata Institute of Fundamental Research, Pune, India, August 2005.

[25] O. E. Kalashev, V. A. Kuzmin, D. V. Semikoz, and G. Sigl, "Ultrahigh-energy neutrino fluxes and their constraints," Physical Review D, vol. 66, no. 6, Article ID 063004, 2002.

[26] A. Y. Neronov and D. V. Semikoz, "Which blazars are neutrino loud?" Physical Review D, vol. 66, no. 12, Article ID 123003, 2002.

[27] O. E. Kalashev, V. A. Kuzmin, D. V. Semikoz, and G. Sigl, "Ultrahigh energy cosmic rays from neutrino emitting acceleration sources?" Physical Review D, vol. 65, no. 10, Article ID 103003 , 2002.

[28] D. Eichler, "High-energy neutrino astronomy-a probe of galactic nuclei," The Astrophysical Journal, vol. 232, pp. 106-112, 1979.

[29] F. Halzen and E. Zas, "Neutrino fluxes from active galaxies: a model-independent estimate," The Astrophysical Journal, vol. 488, no. 2, pp. 669-674, 1997.

[30] S. Adrián-Martínez, I. Al Samarai, A. Albert et al., "Measurement of atmospheric neutrino oscillations with the ANTARES neutrino telescope," Physics Letters B, vol. 714, no. 2-5, pp. 224230, 2012,.

[31] S. Adrián-Martínez, J. A. Aguilar, I. Al Samara et al., "First search for point sources of high energy cosmic neutrinos with the ANTARES neutrino telescope," The Astrophysical Journal Letters, vol. 743, no. 1, article L14, 2011.

[32] M. G. Aartsen, R. Abbasi, Y. Abdou et al., "Measurement of the atmospheric $v_{e}$ flux in IceCube," Physical Review Letters, vol. 110, no. 15, Article ID 151105, 7 pages, 2013.

[33] R. Abbasi, Y. Abdou, T. Abu-Zayyad et al., "Time-integrated searches for point-like sources of neutrinos with the 40-string IceCube detector," The Astrophysical Journal, vol. 732, no. 1, article 18, 16 pages, 2011.

[34] A. V. Avrorin, V. M. Aynutdinov, V. A. Balkanov et al., "Search for high-energy neutrinos in the Baikal neutrino experiment," Astronomy Letters, vol. 35, no. 10, pp. 651-662, 2009.

[35] T. Ebisuzaki, H. Mase, Y. Takizawa, Y. Kawasaki, and K. Shinozaki, "The JEM-EUSO mission," in Proceedings of the 16 International Symposium on Very High Energy Cosmic Ray Interactions ( ISVHECRI '10), Batavia, Ill, USA, June-July 2010.

[36] T. Abu-Zayyad, R. Aida, M. Allen et al., "The cosmic-ray energy spectrum observed with the surface detector of the telescope array experiment," The Astrophysical Journal Letters, vol. 768, no. 1, article L1, 5 pages, 2013.

[37] Pierre Auger Collaboration, P. Abreu, M. Aglietta et al., "Largescale distribution of arrival directions of cosmic rays detected above $10^{18} \mathrm{eV}$ at the Pierre Auger Observatory," The Astrophysical Journal, vol. 203, no. 2, article 34, 20 pages, 2012.

[38] Pierre Auger Collaboration, P. Abreu, M. Aglietta et al., "A search for point sources of EeV neutrons," The Astrophysical Journal, vol. 760, no. 2, article 148, 11 pages, 2012.
[39] J. M. Mazzarella, K. Iwasawa, T. Vavilkin et al., "Investigation of dual active nuclei, outflows, shock-heated gas, and young star clusters in markarian 266," The Astronomical Journal, vol. 144, no. 5 , article 125, 43 pages, 2012.

[40] A. Marconi and L. K. Hunt, "The relation between black hole mass, bulge mass, and near-Infrared luminosity," The Astrophysical Journal Letters, vol. 589, no. 1, pp. L21-L24, 2003.

[41] K. M. Dasyra, L. C. Ho, L. Armus et al., "High-ionization midinfrared lines as black hole mass and bolometric luminosity indicators in active galactic nuclei," The Astrophysical Journal Letters, vol. 674, no. 1, pp. L9-L12, 2008.

[42] J. Houck, T. Roellig, E. Furlan et al., "Mid-infrared spectra of class I protostars in Taurus," The Astrophysical Journal, vol. 154, no. 1, pp. 391-395, 2004.

[43] M. W. Werner, K. I. Uchida, K. Sellgren et al., "New infrared emission features and spectral variations in NGC 7023," The Astrophysical Journal, vol. 154, no. 1, pp. 309-314, 2004.

[44] K. Gebhardt, J. Kormendy, L. C. Ho et al., "Black hole mass estimates from reverberation mapping and from spatially resolved kinematics," The Astrophysical Journal Letters, vol. 543, no. 1, pp. L5-L8, 2000.

[45] L. Ferrarese, R. W. Pogge, B. M. Peterson, D. Merritt, A. Wandel, and C. L. Joseph, "Supermassive black holes in active galactic nuclei. I. The consistency of black hole masses in quiescent and active galaxies," The Astrophysical Journal Letters, vol. 555, no. 2, pp. L79-L82, 2001.

[46] B. M. Peterson, L. Ferrarese, K. M. Gilbert et al., "Central masses and broad-line region sizes of active galactic nuclei. II. A homogeneous analysis of a large reverberation-mapping database," The Astrophysical Journal, vol. 613, no. 2, pp. 682-699, 2004.

[47] N. Konjević, "Plasma broadening and shifting of nonhydrogenic spectral lines: present status and applications," Physics Report, vol. 316, no. 6, pp. 339-401, 1999.

[48] Y. Shen, G. T. Richards, M. A. Strauss et al., "A catalog of Quasar properties from SDSS DR7," The Astrophysical Journal, vol. 194, no. 2, pp. 45-66, 2011.

[49] S. L. Shapiro and S. A. Teukolsky, Black Holes, White Dwarfs, and Neutron Stars, Wiley-Interscience, New York, NY, USA, 1983.

[50] B. L. Friman and O. V. Maxwell, "Neutrino emissivities of neutron stars," The Astrophysical Journal, vol. 232, pp. 541-557, 1979.

[51] O. V. Maxwell, G. E. Brown, D. K. Campbell, R. F. Dashen, and J. T. Manassah, "Beta decay of pion condensates as a cooling mechanism for neutron stars," The Astrophysical Journal, vol. 216, pp. 77-85, 1977.

[52] N. Iwamoto, "Quark beta decay and the cooling of neutron stars," Physical Review Letters, vol. 44, no. 24, pp. 1637-1640, 1980.

[53] A. Burrows, "Beta decay in quark stars," Physical Review Letters, vol. 44, no. 24, pp. 1640-1643, 1980. 

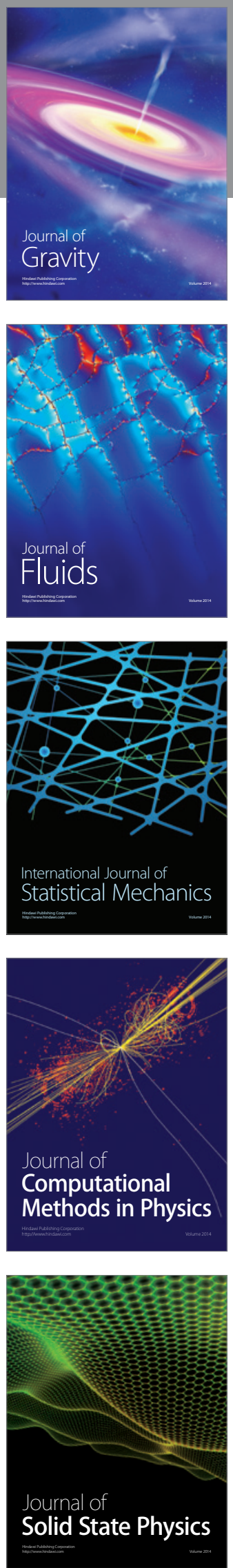

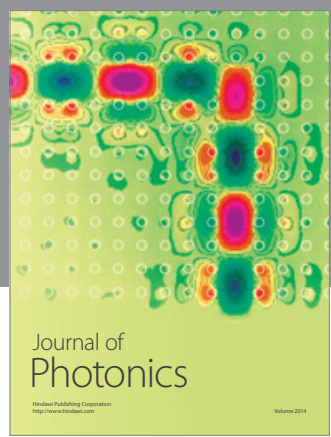

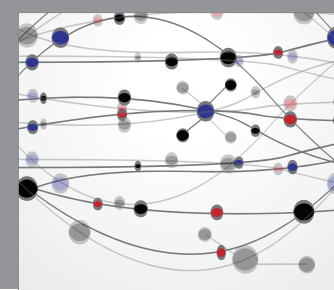

The Scientific World Journal

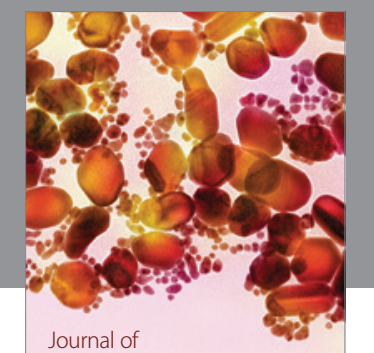

Soft Matter
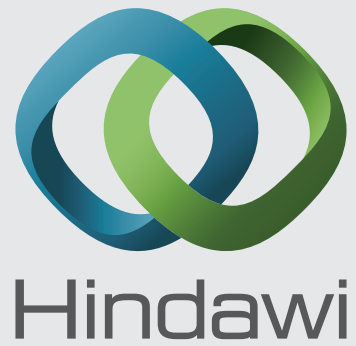

Submit your manuscripts at

http://www.hindawi.com
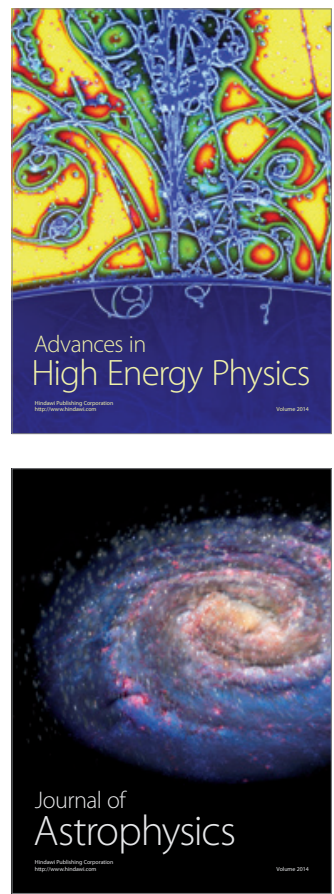
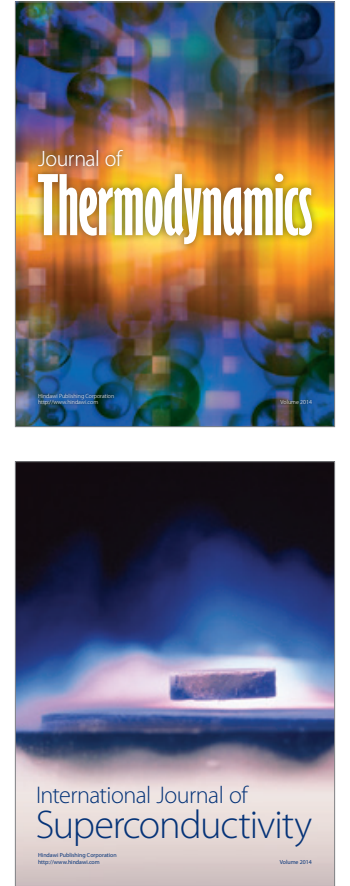
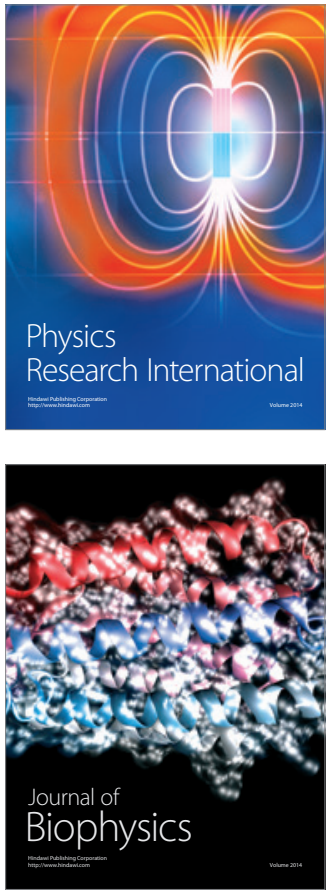
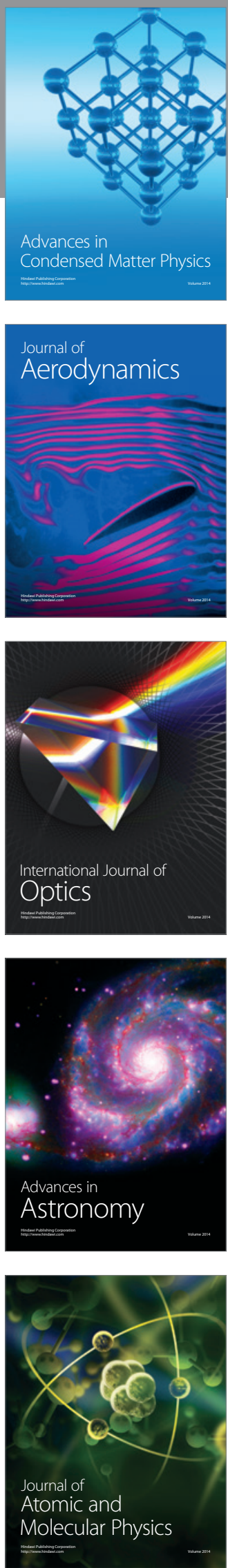